\title{
Is the glycaemic index of carbohydrates relevant to enhanced performance in young athletes?
}

\author{
M. Westlake and M. Hawkins \\ School of Science, Technology and Health, University Campus Suffolk, Ipswich, UK
}

\begin{abstract}
Nutritional guidance for the adolescent athlete engaged in demanding training schedules is mostly based on adult recommendations ${ }^{(1)}$. The young athlete's ultimate aim is to maintain levels of carbohydrate required by the body during prolonged intensive training as this is a key factor in performance and exercise capacity. Although paediatric exercise science literature is revealing increasing differentiations in exercise physiology between the child and adult athlete, there remains little child-specific evidence regarding the regulatory mechanisms of carbohydrates during exercise ${ }^{(2)}$. The aim of this study was to examine whether carbohydrate (CHO) of different glycaemic index (GI) would enhance performance in adolescent swimmers when used as the carbohydrate supplementation indicator in a pre-exercise meal. Eleven adolescent swimmers (mean (SD) age: 12.9 (SD 1.24) completed two trials, separated by $14 \mathrm{~d}$, in a random crossover design. Each consumed a low GI (pasta) or high GI (potato) evening meal $(2.5 \mathrm{~g} \mathrm{CHO} / \mathrm{kg}$ body mass) 90 min prior to initial exercise followed by $50 \mathrm{~m}$ and $400 \mathrm{~m}$ front crawl time trials. Dietary intake was also controlled from lunchtime onwards on each trial day.
\end{abstract}

\begin{tabular}{lcc}
\hline Variable & Performance time (s) & Performance time (s) \\
& $50 \mathrm{~m}$ front crawl & $400 \mathrm{~m}$ front crawl \\
\hline Low GI test & 32.87 (SD 1.92) & 327.11 (SD 14.55) \\
High GI test & 32.83 (SD 1.26) & 323.95 (SD 15.0) \\
$P$ (2-tail) & 0.92 & 0.17 \\
\hline
\end{tabular}

No statistically significant differences were observed in performance times in the low GI trial $v$. high GI trial in either $50 \mathrm{~m}$ [mean (SD) low $v$. high GI $50 \mathrm{~m}: 32.87$ (SD 1.92) v. 32.83 (SD 1.26) or 400 m (mean (SD) low $v$. high GI 400 m: 327.11 (SD 14.55) v. 323.95 (SD 15.0)] $(P>0.05)$. The results of this study indicate that there was no significant improvement in either sprint or endurance performance times, despite a greater dependence on $\mathrm{CHO}$ with this mode of exercise, following CHO supplementation of different GI. Due to ethical limitations, it was not possible to obtain blood samples or expired gas samples from the adolescent subjects, at any point during this study. This would have enabled the measurement and observation of certain metabolic responses and subsequent substrate utilisation to the high GI and low GI meals, and their influences in relation to exercise performance. The unique fuel-use profile and blood-lactate concentration of a child athlete during submaximal exercise has mostly been attributed to their increased oxidative and decreased glycolytic ability compared with adults. However, this view has recently been challenged by some eminent researchers ${ }^{(3)}$. It is clear that further research is required to investigate any potential performance benefits to the adolescent athlete, using the GI as the CHO supplementation indicator in a pre-exercise meal.

1. Aucouturier J, Baker J \& Duche P (2008) Sports Med 38, 213-238.

2. Burke L, Collier G \& Hargreaves M (1998) Int J Sports Nutr 8, 401-415.

3. Timmons B, Bar-Or O \& Riddell M (2007b) J Appl Phys 103, 995-1000. 